\title{
Displaced Soundscapes: A Survey of Network Systems for Music and Sonic Art Creation
}

\author{
Álvaro Barbosa
}

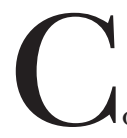

ollaboration has long been a key element in music; therefore, the use of collaborative systems based on computer networks to achieve musical results were a natural development. The advent of computer network music dates back to the late 1970 s with early experimental performances in California by the League of Automatic Music Composers [1]. Up until the early 1990s, systems for musical collaboration using computers were based on local networks. In the last decade, the massive worldwide growth of the Internet has increased the possibilities for composers, performers and audiences. However, it is well known that the phenomenon of network delay is a major drawback for real-time collaboration over global networks. At the global level, physical limitations in current network technology introduce higher latency than is acceptable for real-time acoustic collaboration.

For the human ear to perceive two sounds as simultaneous, the sounds should not be displaced in time more than $20 \mathrm{msec}$ [2], which means that for mutual awareness to be supported in a bilateral performance, the maximum threshold would be around $40 \mathrm{msec}$ (the time it would take a performer to perceive a second performer's reaction to his or her action). It should be noted that the perception of two different sounds performed simultaneously is strongly dependent on sound characteristics (timbre, pitch and loudness), musical style and other types of feedback such as visual or physical stimuli. Nevertheless, a 20-msec threshold should be adequate.

If we consider the smallest possible peer-to-peer connection between two opposite points on the planet, we have an approximate distance of $20,004.5 \mathrm{~km}$ (half the distance of earth's

Fig. 1. Screen shot of the FMOL software bamboo interface. With this interface the user can manipulate and generate individual or collaborative pieces. (@ Sergi Jordà)

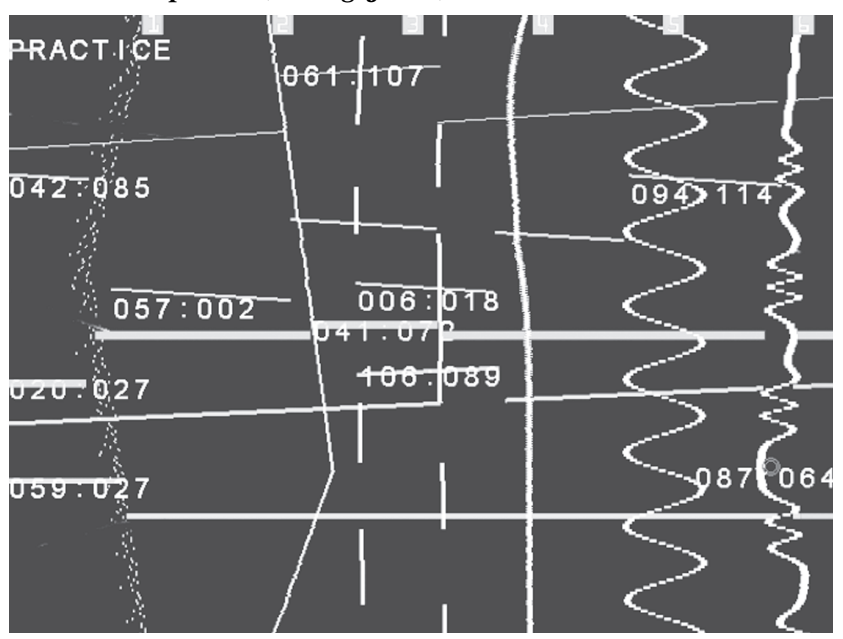

perimeter: 40,009 km). Even with data transfer at the speed of light (approximately 300,000 km per sec) and unlimited bandwidth, bidirectional latency would reach approximately $133.4 \mathrm{msec}$, which is much higher than the tolerable threshold. With such numbers in mind, it is not surprising that network delay is an assumed part of creating music on-line.

The thought that network delay is particular to the medium and that composers should embrace this fact when creating music is clearly expressed by experimental artist Atau Tanaka in an interview with Golo Föllmer:

I find Internet time delay rather interesting and I think of it as a kind of unique acoustic of this media.... [R]ather than to play existing music on this new time basis, what is interesting to me is trying to find a musical language that works on this time axis.... [3]

This same idea motivated the SoundWIRE group at the Center for Research in Music and Acoustics (CCRMA) at Stanford University to conduct significant research over the last few years to address the influence of network conditions in acoustic communication $[4,5]$.

Latency has a highly variable and unpredictable nature, creating time-based errors, de-sequencing and even partial loss of content, resulting in difficult conditions for performance control. There are major efforts under way to improve the situation by increasing bandwidth and data compression options. Such improvements, along with restricting projects to Large Area Networks (LANs) or to Wide Area Networks (WANs) in geographically constrained territories (e.g. a country or even a continent), may reduce network latency to levels that will not impede real-time acoustic communication over the Internet. Network latency tends to be central to the discussion of network-based activities, yet there are other important issues, such as who will perform with these systems and whether timebased musical events/performances are always appropriate.

\section{Redefining The AcoustiC Community}

Designing and implementing a network music system supposes that new, meaningful sonic results can be achieved by collab-

Álvaro Barbosa (researcher), Music Technology Group, Pompeu Fabra University, Passeig de Circumval·lació 8, 08003 Barcelona, Spain. E-mail: < abarbosa@iua.upf.es>. 
oration over computer networks. When first addressing this supposition, one should start by considering the target audience. In 1984 Barry Truax introduced the concept of the acoustic community in his book Acoustic Communication:

The "Acoustic Community" may be defined as any soundscape in which acoustic information plays a pervasive role in the lives of the inhabitants. ... Therefore the boundary of the community is arbitrary and may be as small as a room of people, a home or a building, or as large as an urban community, a broadcast area or any other system of electroacoustic communication [6].

Truax's concept of the acoustic community can be viewed from an on-line context. The notion of a soundscape familiar to all users is inevitably tied to sonic events transmitted and produced with computers, suggesting that computergenerated electronic music might be an engaging format to address in the place of more traditional musical formats. However, traditional musical culture is somewhat strict regarding what constitutes a music event, and one of the major questions regarding collective music creation by indiscriminate Internet users is whether this community is prepared to express meaningful musical results. The fact that Internet technology provides permanent public access to music creation systems and listening paradigms raises additional questions regarding traditional aspects of a musical piece, such as its duration. An ongoing musical piece that is not constrained by time limits seems to be an appropriate form for using some of the emerging systems discussed later in this article.

\section{TIME Dimensions OF a Permanent Event}

In the book Microsounds, Curtis Roads introduces a taxonomy of timescales from a music theory perspective [7]. In this proposal, music timescales are, in decreasing order, the Infinite timescale, the Supra timescale, the Macro timescale, the Meso timescale, the Sound Object timescale, the Micro timescale, the Sample timescale, the Subsample timescale and the Infinitesimal timescale.

Most music creations driven by an event are in the Macro timescale, defined by Roads as "the timescale of overall music architecture or form, measured in minutes or hours, or in extreme cases, days" [8]. However, one might wonder where an ongoing musical piece, permanently available for use by hybrid communities of creators and listeners, would

belong. Realistically it should fit in the Supra timescale, defined as a "timescale beyond that of an individual composition and extending into months, years, decades, and centuries" [9], since the Infinite timescale is an abstraction beyond the time life of the present cultural and technological state of development.

Some recent artistic proposals approach unlimited music events. In 2000 Antoine Schmitt created the Infinite $C D$ for Unlimited Music [10]. Once inserted in a computer, this CD generates music infinitely, always different yet always similar, with no images or form of interaction. Another approach to this idea explores Internet technology and user interaction: In 2003 Chris Brown presented the Eternal Network Music project, a flexible piece created on-line that allows up to four players at a time to change the harmony and motion of eight modulated sine-wave oscillators to produce a richly vibrating drone that continues endlessly [11].

\section{NETWORK SYSTEMS FOR MUSIC AND SONIC ARTS}

Given the subset of the musical universe constrained to computer-network-based music, it is useful to consider Guy E. Garnett's definition of music:

\begin{abstract}
The nature of music, particularly in the century of John Cage, multiculturalism, and other varieties of aesthetic choice, becomes more problematic. Nonetheless, I think it is possible to reduce the problem somewhat. Just as I have considered aesthetics in only its broadest manifestations, similarly, music can be roughly considered to be sounds made with aesthetical intent, or even sound listened to with aesthetic interest. The former gives more weight to the role of the creator, while the latter formulation tends to privilege the listener [12].
\end{abstract}

In this sense we can consider the sonic outcome of a collective Internet creation as a music event, since users commit themselves to create a sound piece collaboratively with aesthetic intent and simultaneously are audience members interpreting the results with aesthetic interest.

Computer network music falls in part within the larger category of sonic art, a term that emerged in the 1960 s and that applies to a very broad spectrum of scenarios in which acoustic phenomena are manipulated in different ways. Historically, sonic art came from the academic tradition of electroacoustic music, as until quite recently, advanced electronics and computer technology for audio were available only to members of insti- tutions such as universities and radio stations. The electroacoustic music tradition dates back to the 1950s and 1960s, when it emerged as a discipline in college and university music departments, based on the work of composers such as Pierre Schaeffer and Karlheinz Stockhausen. Although there is no comprehensive definition of sonic art, in the 1980s this field became a playground of diversified artistic proposals and experiments with electronic and digital music creation when computer technologies became more widely available to the ordinary music creator, and expanded in the 1990s with the advent of Internet-based communication.

\section{EARLY EXPERIMENTS WITH MUSIC AND COMPUTER NETWORKS}

In the late 1970 s the commercialization of personal computers in the United States, which allowed the fine-tuning of network topologies, enabled the first attempts by experimental musicians to create local computer networks. The first events using computer networks to create collaborative music were performed by the Oakland, California, group the League of Automatic Music Composers [13]. The "League" came together through the mutual interests of central protagonists Jim Horton, John Bischoff and Rich Gold, who named their new genre of musical performance Network Computer Music.

In 1985 the Network Muse, a network music festival, was held in San Francisco, California, and featured a collective of electronic musicians such as John Bischoff, Tim Perkis, Chris Brown, Mark Trayle, Scot Gresham-Lancaster, Larry Polansky, Phil Stone and Phil Burk. From the context created around the activity of these composers, the League evolved into a subsequent group in 1987, the Hub, which employed more accurate communication schemes by using the MIDI protocol [14].

\section{LOCAL MUSICAL NeTWORKS}

In 2002 Gil Weinberg introduced the concept of Interconnected Musical Networks (IMNs) with an example from a performance at the MIT Media Lab in the spring of 1998 :

[T] wo musicians were playing a newly developed multiplayer squeezable instrument. While controlling the pitch curve of his own part, one of the players was also continuously manipulating the other player's timbre. This manipulation 
led the second player to modify her play gestures in response to the new timbre she received from her peer. ... [A]n immersive and interdependent network of subtle mutual influences emerged, leading the two performers to a unique playing experience, where unpredictability, dynamism, self-evolving musical behaviours, and interdependent collaboration are at the core [15].

The concept of a musical instrument designed to be played simultaneously by more than one person is not new, but there are very few examples in the history of Western music, other than the piano. With local high-speed computer networks and sensor technology, a new universe of possibilities has been unveiled in this field, providing a context for new experiments such as the Jam-OWorld multi-player music controller, an interactive drumming table that allows groups of people with or without previous musical knowledge to share audiovisual experiences through its integration of interactive music elements with realtime video and computer graphics projections in a collaborative environment $[16,17]$.

However, some single-user instruments that are not designed to be played simultaneously by several performers may instead be able to evoke several instances of themselves, allowing different users to play simultaneously. Furthermore, the idea of a multi-user instrument does not necessarily require performance interdependency, as explained by Weinberg above [18]. With IMNs, in order to achieve virtuosic results, it is critical to have a real-time network system, due to the interdependency required among performers. Therefore, this approach is mostly constrained to local networks.

\section{COMPOSITION SUPPORT SYSTEMS}

Two or more composers can utilize various techniques to compose together. Traditionally, a composer conceives the music individually and registers his ideas in symbolic musical notation (a score). Cooperation with other composers in a co-authored piece requires the exchange of ideas among contributors by a bilateral analysis of each participants' additions to the score. An early system based on the idea of using Internet communication to enhance traditional joint composition appeared in the early 1990s with Craig R. Latta's NetJam [19]. This system allowed a community of users to collaborate in producing music in an asynchronous way by exchanging MIDI files through e-mail.
With the advent of recording and nonlinear editing technologies, a new way to compose music emerged. The use of recording studios as compositional tools has become increasingly common, especially in producing popular music. In a recording studio one or more musicians can record their instrumental performances (synchronously or asynchronously), resulting in raw material recorded acoustically that can be manipulated to a great degree to create a complete musical piece. Due to its ease of use, this method is highly suitable for musicians and composers who are not formally trained, since it reduces the temporal gap between the spark of an idea and witnessing the result, and therefore provides the possibility to react to, transform and improvise a piece more quickly and efficiently.

For Internet users interested in music, a new class of Internet applications has emerged that enables the idea of the online recording studio. This concept has been realized in the form of distributed systems coupled with centralized servers that manage multiple sessions and groups of users. In these systems the interface resembles typical nonlinear multitrack editing software and allows users to lay down MIDI and digital audio tracks, in either an asynchronous or synchronous non-real-time mode, collaborating with other users from the same session. Well-known applications in this area are the ResRocket Surfer [20] and Tonos systems [21], which received support from music industry manufacturers.

Fig. 2. Screen shot of the FMOL web-based tree-structured database with multiple-generation pieces composed by different users. The users can upload their FMOL pieces to this server, making them available for other users to modify. (๔ Sergi Jordà)

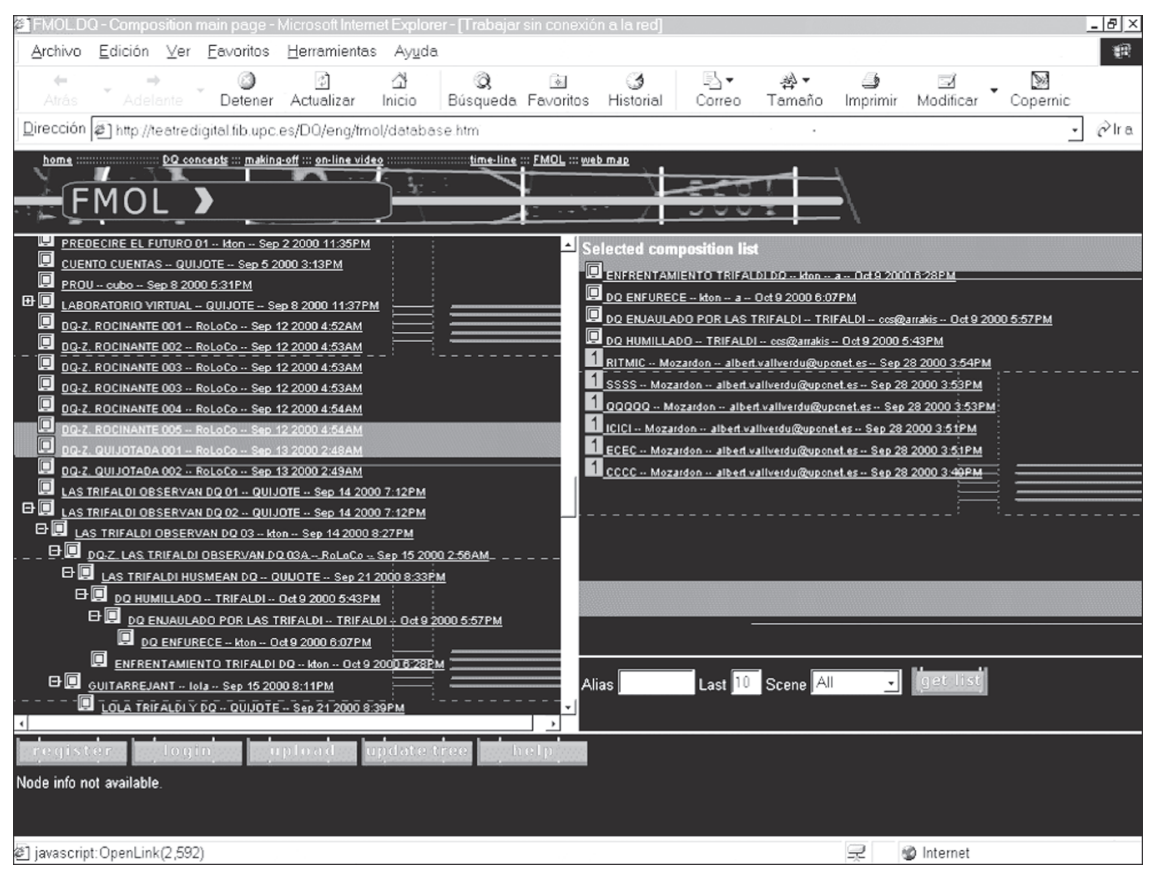

A different approach to reaching a broad spectrum of users is to publish specific software tools via the Internet that either are dependent on special-purpose hardware or are proprietary experimental systems from companies or research groups. In 1995, with support from Sun Microsystems, the Institut de Recherche et Coordination Acoustique/Musique (IRCAM) started an on-line studio [22] based on client/server web technology. This project aimed to provide access to some of IRCAM's sound databases and sophisticated sound-processing tools such as the Super Phase Vocoder (SVP). This project has recently developed the On-Line Sound Palette under the CUIDADO project framework [23]. A similar project was started in 1997 by Ramon Loureiro at the Audiovisual Institute of Pompeu Fabra University in Barcelona, Spain [24]. This system made it possible to have a web front end to Xavier Serra's Spectral Modelling Synthesis technique [25].

\section{Collective Creation SYSTEMS}

Composition can be approached through a community perspective, since the Internet makes it possible to create systems available to large communities of anonymous users. In 1997 the Catalan theater company La Fura dels Baus commissioned experimental artist Sergi Jordà to develop an on-line collective music creation system. The system allows the par- 
ticipation of Internet users in the creation of musical pieces that were included later in the playF@ust 3.0, freely inspired by Goethe's work. The original system followed a client/server model, allowing composers, using the Faust Music OnLine (FMOL) software, to log into a central web-based server to download and upload pieces stored in a song treestructure database (Figs 1 and 2) [26]. During the periods that this project was on-line, January-March 1998 and September-October 2000, several hundred composers participated in the creation of excerpts for musical scores for two plays by La Fura and a collective CD.

In 1996 a similar approach, but within a different context, was presented in Tod Machover's Brain Opera, which among other things was an attempt to combine a staged installation and on-line interaction [27]. For this project a series of hyperinstruments were created for use at a physical installation site and through interactive sound applications available on-line. The results from the hyperinstrument performances at the installation, along with results from on-line users, converged in a final performance onstage.

Another project based on the idea of collective music creation, but with a different approach for a live event, was William Duckworth's 1997 Cathedral [28]. This project was one of the first interactive music works created specifically for the World Wide Web. Live events conveying pieces created by users in this environment were broadcast on-line.

\section{TELEPRESENCE SySTEMS}

The idea of presenting performances that include one or more performers at remote sites located anywhere in the world, participating in events taking place in front of live audiences during fixed periods of time, is an exciting one. Logistical considerations must be made for events occurring simultaneously in different time zones, but the main concern in such a setting is usually network latency. One approach to this problem is the use of communications technologies such as high-speed and broadband networks combined with streaming techniques.

A unique public event that occurred on 26 September 1999 illustrates this approach. A musical performance at McGill University in Montreal, Canada, was transmitted to an audience at New York University over the Internet during the 107th Audio Engineering Society (AES) Convention [29]. What made this event distinctive was the audience's reception of an uninterrupted transmission of multichannel audio over a custom system employing both Transmission Control Protocols (TCP) and User Datagram Protocols (UDP), providing its own buffering and retransmission algorithms.

Another project, developed in 1997 in collaboration among the University of Geneva, Switzerland; GRAME, Centre National De Création Musicale, in Lyon, France; and GMD, National Research Center for Information Technology and the Fraunhofer-Gesellschaft, in Birlinghoven, Germany, was based on the development of a telepresence system (unilateral participation), with the goal of creating an environment for distributed rehearsals where the conductor of an orchestra could be present remotely [30]. To give participants the impression of the presence of the conductor in the room and of his awareness of the musicians' gestures, eye contact and bodily expressions, the project required specialpurpose technology with high-quality video and spatialized sound.

A different approach to performance in a live public event is to create systems based on low-cost public-domain technology. Different styles of music, instruments and technical setups have been tried, such as the telemusic and piano master classes of John Young and Randall Packer and New York University's Cassandra Project [31,32].

Fig. 3. The Public Sound Objects system architecture. Several remote clients can simultaneously manipulate sound objects from the central server. The connection from the clients to the server is based on discrete commands issued by the users, and that from the server to the clients is based on a digital audio stream that conveys the performance of all on-line users. (๔) Álvaro Barbosa)

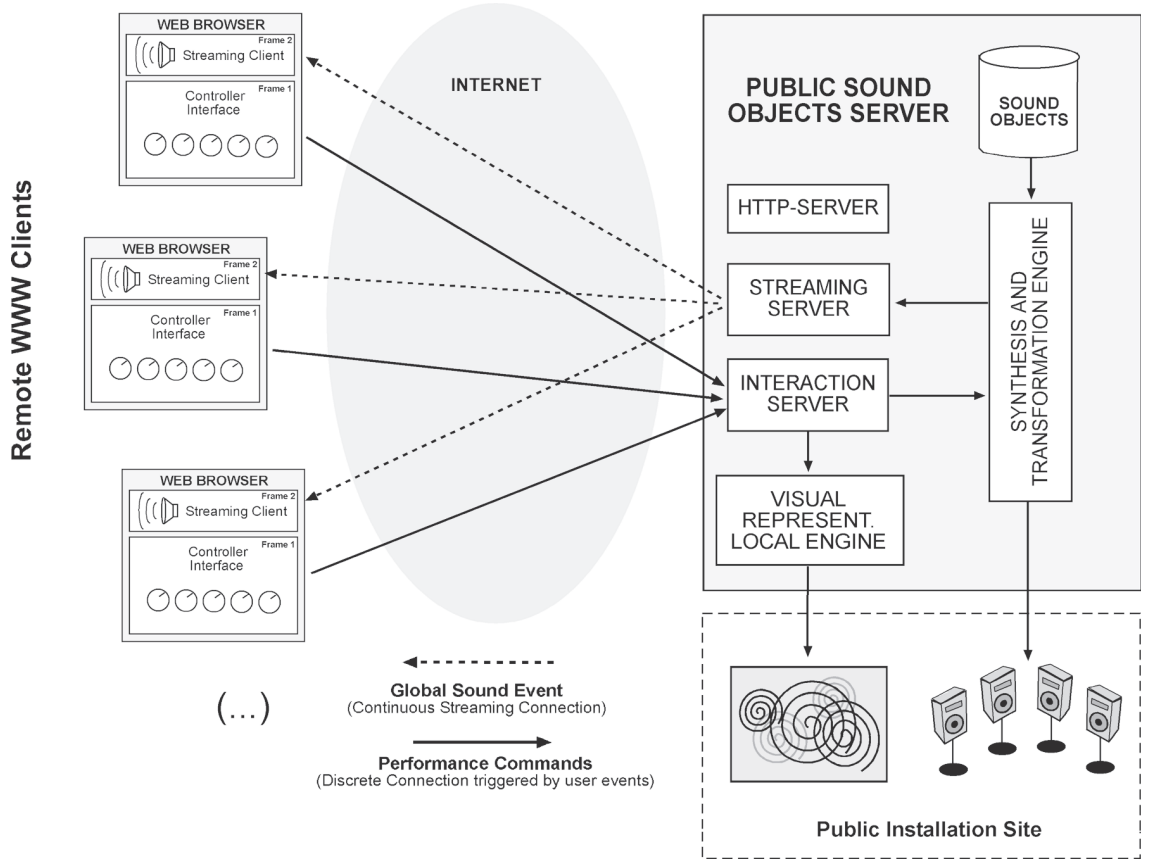


beyond the MIDI format by supporting low-fidelity digital audio [35]. A different approach to this concept based on a mixed environment of an interactive museum installation and synchronous multiuser Internet-based collaboration is the Worldbeat/GlobeMusic project currently hosted by the Ars Electronica Center in Linz, Austria [36]. The creators of this project are developing ways for offline/on-line interaction with other musicians.

An additional experiment that reflects the potential of multi-user remote performance over the Internet took place during the 2001 Networkshop festival in Dresden, Germany, when several collaborative on-line concerts based on Sergi Jordà's FMOL Virtual Music Instrument were presented between Dresden and Barcelona [37]. Even though latency was in the range of $100 \mathrm{~ms}$ with 56 -kb modem connections, the performers conveyed a strong sense of control and playability. The sound-sequencing technique used in this system, based on low-frequency oscillators that excite sound generators, created rhythmic and melodic progressions that supported flexible reaction times and short periods of asynchronicity, motivated by network latency, from the performing partners.

\section{ON-LINE IMPROVISATION AND SHARED SONIC ENVIRONMENTS}

Even though set-up requirements for nonimprovisational performance and for pure improvisation (i.e. "jam" sessions) are physically the same, there are major conceptual differences between these two approaches. In the context presented in this article, nonimprovisational performance means the process of playing a sequence of music events (a score) while providing some sort of synchronism with other music or visual events. In a performance there is space left for individual expression and for improvisation, but in musical terms the event should be driven to a certain extent by a predetermined and rehearsed composition. With pure improvisation, musicians are not engaged in such a systematic approach, leaving more space available for spontaneity, free expression and continuous development of elaborated interactive relationships between the participants.

Since no musical knowledge or instrumental performance requirements can be demanded from an Internet user, a spontaneous improvisational approach is quite suitable; for such an approach an emergent new class of applications en- ables what can be termed shared sonic environments. In such openly shared spaces members of the on-line community can participate in a public event by manipulating or transforming sounds and musical structures or by simply listening to music created collectively.

One of the first examples of such systems is the WebDrum, a drum box that can be shared by several people over the Software Synthesis API for Java JSyn and the TransJam Architecture [38]. Another recent artistic proposal following this approach is Atau Tanaka's $M P 3 Q$ piece, classified by the author as a shared online sound space that streams multiple channels of MP3 audio from different servers [39]. Users can concurrently manipulate these MP3 sources by using a 3D cube to actuate a graphic representation of the system's current behavior.

Public Sound Objects is a system designed from scratch as a shared sonic space by the Music Technology Group at Pompeu Fabra University [40]. In this project the concept of a shared space is explored simultaneously by communitydriven sonic creation and an onsite audiovisual installation, bringing together a physical space and a virtual presence on the Internet. Public Sound Objects are based on classic client/server architecture. The server handles the actual sound-synthesis computation, and the interaction interface is implemented on the client side. One of the main characteristics of this implementation is its modularity (Fig. 3). It is important to note that the user to synthesis server does not Internet, based on Phil Burk's Audio

require a continuous real-time input stream. Rather, it is based on a communication model that enables triggering of sounds on the server side in response to discrete events on the client side, providing faster communication from the client to the server, based on the transmission of small text messages.

\section{Cllassification OF COMPUTER-SUPPORTED Collaborative MUSiC}

The systems I have presented in this article each have different sonic communication requirements; therefore, I have categorized them into different classes:

- Local Interconnected Musical Networks-used in organized events for groups of performers who interact in real time with a set of musical instruments (or virtual musical instruments) with sonic interdependency provided by a local computer network.

- Musical Composition Support Systems-used to assist more traditional forms of musical composition and production, both for composition based on written music supports and music production based on experimental processes in recording studios. Musical Composition Support Systems enhance the traditional collaboration paradigms by allowing geographical displacement and asynchronous collaboration.

- Remote Music Performance Systems-used in organized events for groups of multiple remote perform-

Fig. 4. A classification space for computer-supported collaborative music. In this diagram one can compare the different categories of a network music system in terms of the user's location and performance synchronicity. (@) Álvaro Barbosa)

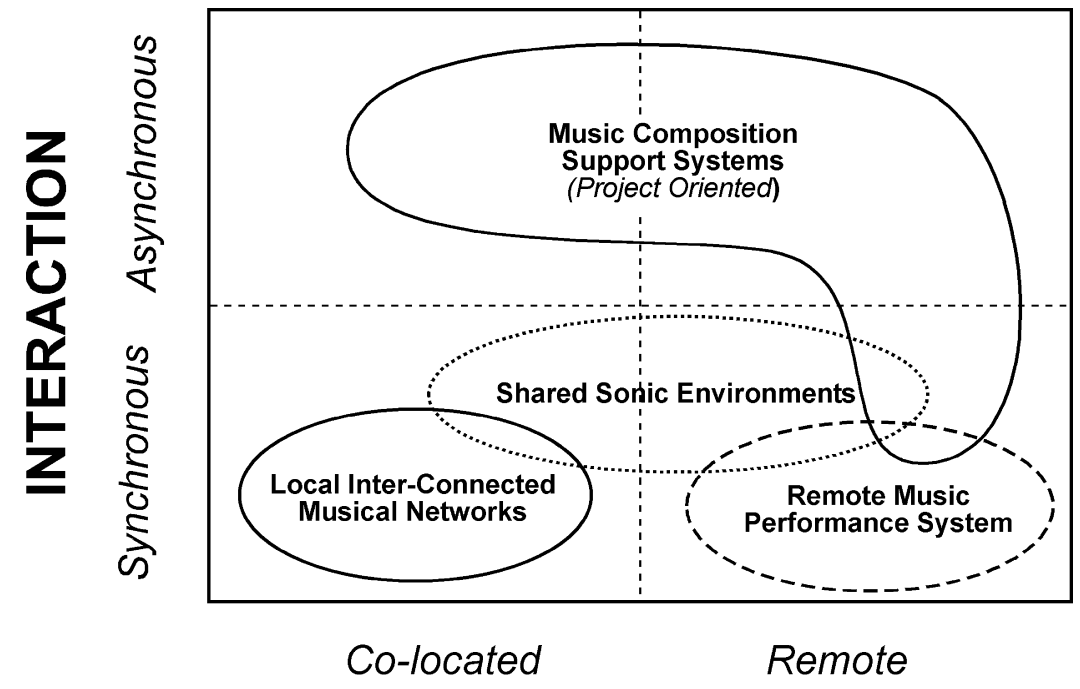

LOCATION 
ers/users, displaced in space, improvising and interacting synchronously with a set of musical instruments (or virtual musical instruments). In this case sonic interdependency is affected by network latency. Telepresence (remote unilateral participation) is a particular case of this set of applications.

- Shared Sonic Environments-a new class of emerging applications that explores the Internet's distributed and shared nature. They are not oriented towards time-limited event scenarios, and their use is more suitable for synchronous improvisation. Since these systems are addressed to broad audiences, previous musical knowledge from the participants usually is not required, and therefore results often are experimental sonic pieces.

Based on these categorizations, a classification space can be defined taking into account the environmental facets: synchronous and asynchronous for the time dimension; remote and co-located for the space dimension (Fig. 4). This categorization of sonic art systems is inspired by Tom Rodden's proposal for a classification space, introduced in 1997 for Computer-Supported Cooperative Work (CSCW) [41], a field in computer science that represents a major focus area of the Special Interest Group on Groupware (SIGGROUP), of the educational and scientific computing society the Association for Computing Machinery (ACM).

It should be noted that these are by no means closed categories, and many of the applications belong to more than one class. For instance, the FMOL system described earlier represents an extreme example of an application with characteristics from various categories. It can be used locally in an interconnected music network; it is a multi-user instrument; it can be used in remote music performance over the Internet; it supports online asynchronous collective composition in a sort of centralized studio paradigm.

\section{Conclusions}

This study is not exhaustive, and owing to the experimental nature of this field we can assume that information about many interesting and relevant projects has not yet been published or made public. Nevertheless, I have attempted to provide here a variety of representative projects to provide useful references and concepts for future work on network music systems.
One may infer from this study that most of the projects approaching geographical displacement over the Internet are oriented towards:

1. The creation of networks where documents in digital audio or logical formats can be exchanged among geographically displaced contributors, in project-oriented collaboration paradigms such as in a typical computer-supported cooperative work application

2. Providing a channel to allow telepresence between performative spaces and therefore enhancing the efficiency of traditional collaborative paradigms for musical performance/composition, musical education or even for music sharing, by adding long-distance collaboration possibilities

3. The creation of community-oriented shared virtual environments, where users dynamically join and leave, supporting collaborative ongoing sonic performance based on the simple manipulation of sound objects in a soundscape or on the creation of musical structures. This approach goes beyond the enhancements of existing acoustic communication paradigms, focusing on diverse Internet collaboration.

Just as similar paradigms oriented towards visual or textual communication (Multi User Dungeons [MUDs], Object Oriented MUDs [MOOs], Internet Relay Chats [IRC], Active Worlds, etc.) tend to lead to new mechanisms of interaction not usually seen in "real life" [42], a similar result can be expected in paradigms oriented towards music or sonic art, suggesting that the sonic outcome of such systems could express interesting new artistic results. It is clear that this area of sonic creation is quite promising, not only from the fact that it allows the enhancement of known paradigms to make music, but also because it provides a context for stylistic novelty.

\section{Acknowledgments}

I would like to thank Xavier Serra, Sergi Jordà, Perfecto Herrera and Martin Kaltenbrunner for their support and contribution to this study. This work was supported by the Portuguese institution Fundação para a Ciência e Tecnologia (Foundation for Science and Technology).

\section{References and Notes}

1. J. Bischoff, R. Gold and J. Horton, "Music for an Interactive Network of Microcomputers," Computer Music Journal 2, No. 3, 24-29 (1978).

2. I. Hirsh, "Auditory Perception of Temporal Order," Journal of the Acoustical Society of America 31, No. 6, 759-767 (1959).
3. G. Föllmer, Crossfade-Sound Travels on the WebSoft Music, a joint project of San Francisco Museum of Modern Art, San Francisco, California; ZKMCenter for Art and Media, Karlsruhe, Germany; Walker Art Center, Minneapolis, Minnesota; Goethe Forum, Munich, Germany; 2001. See <http:// crossfade.walkerart.org/>.

4. C. Chafe, S. Wilson, R. Leistikow, D. Chisholm and G. Scavone, "Simplified Approach to High Quality Music and Sound over IP," in Proceedings of the Digital Audio Effects (DAFX) Conference (COSTG6 European Cooperation in the field of Scientific and Technical Research Framework, 2000) pp. 159-164.

5. C. Chafe and R. Leistikow, "Levels of Temporal Resolution in Sonification of Network Performance," in Proceedings of the International Conference on Auditory Display (Helsinki, Finland: Helsinki Univ. of Technology, 2001), pp. 50-55.

6. B. Truax, Acoustic Communication (Norwood, NJ: Ablex, 1984)

7. C. Roads, Microsounds (Cambridge, MA: MIT Press, 2001).

8. Roads [7] pp. 2-3.

9. Roads [7] p. 3.

10. A. Schmitt and V. Epplay, Infinite CD for Unlimited Music (2000). See < http://www.infiniteCD.org/>.

11. C. Brown and J. Bischoff, Eternal Network Music (2003) < http://crossfade.walkerart.org/brown bischoff2/eternal/ $>$

12. G. Garnett, "The Aesthetics of Interactive Computer Music," Computer Music Journal 25, No. 1, 21-33 (2001).

13. Bischoff, Gold and Horton [1].

14. S. Gresham-Lancaster, "The Aesthetics and History of the Hub: The Effects of Changing Technology on Network Computer Music," Leonardo Music Journal 8 (1998) pp. 39-44.

15. G. Weinberg, "Interconnected Musical Networks: Bringing Expression and Thoughtfulness to Collaborative Music Making," thesis proposal, MIT Media Laboratory, Cambridge, MA, $2002<$ http://web. media.mit.edu/ gili/publications/Phd_proposal\% 20-\%20IMN\%20Final.pdf>.

16. T. Blaine and C. Forlines, "JAM-O-WORLD: Evolution of the Jam-O-Drum Multi-Player Musical Controller into Jam-O-Whirl Gaming Interface," in Conference on New Interfaces for Musical Expression (NIME) (2002) pp. 17-22.

17. The JAM-O-WORLD web site $<$ http://www.etc. cmu.edu/projects/jamoworld/ $>$

18. Weinberg [15].

19. C. Latta, "Notes from the NetJam Project," Leonardo Music Journal 1 (1991) pp. 103-105.

20. M. Moller, W. Henshall, T. Bran and C. Becker, The ResRocket Surfer - Rocket Network (1994) < http:/ / www.rocketnetwork.com/>.

21. For more information about Tonos, see $<$ http://www.tonos.com $>$.

22. R. Wöhrmann and G. Ballet, "Design and Architecture of Distributed Sound Processing Systems for Web-Based Computer Music Applications," Computer Music Journal 23, No. 3, 73-84 (2002).

23. H. Vinet, P. Herrera and F. Pachet, "The CUIDADO Project," in Proceedings of ISMIR 2002, 3rd International Conference on Music Information Retrieval (Paris, France: IRCAM, 2002).

24. R. Loureiro and X. Serra, "A Web Interface for a Sound Database and Processing System," in Proceedings of the International Computer Music Conference (ICMC, 1997) pp. 360-363.

25. X. Serra, "Musical Sound Modelling with Sinusoids plus Noise," in G.D. Poli, A. Picialli, S.T. Pope 
and C. Roads, eds., Musical Signal Processing (the Netherlands: Swets \& Zeitlinger, 1997) part 1, chap. 3.

26. S. Jordà, "Faust Music On Line (FMOL): An Approach to Real-Time Collective Composition on the Internet," Leonardo Music Journal 9 (1999) pp. 5-12.

27. R. Rowe, Machine Musicianship (Cambridge, MA: MIT Press, 2001) pp. 360-362.

28. W. Duckworth, "Making Music on the Web," Leonardo Music Journal 9 (1999) pp. 13-17.

29. A. Xu, W. Woszczyk, Z. Settel, B. Pennycook, R Rowe, P. Galenter, J. Bary, M. Geoff, J. Corey and J. Cooperstock, "Real-Time Streaming of Multichannel Audio Data over the Internet," Journal of the Audio Engineering Society 47, No. 11 (New York, NY: AES, 2000) p. 1,022 .

30. D. Konstantas, "Overview of a Telepresence Environment for Distributed Musical Rehearsals," Proceedings of the ACM Symposium on Applied Computing (Atlanta, GA: ACM, 1998) pp. 135-137.

31. J. Young and I. Fujinaga, "Piano Master Classes via the Internet," in Proceedings of the International Computer Music Conference (ICMC, 1999) pp. 135-137.

32. D. Ghezzo, J. Gilbert, A. Smith and S. Jacobson, The Cassandra Project (1996). For more information, see <http://www.nyu.edu/pages/ngc/ipg/cassandra/>

33. B. Bongers, "An Interview with Sensorband," Computer Music Journal 22, No. 1 (1998) pp. 13-24.
34. D. Gang, V. Chockler, T. Anker, A. Kremer and T. Winkler, "TransMIDI: A System for MIDI Sessions Over the Network Using Transis," in Proceedings of the International Computer Music Conference (ICMC, 1997) pp. 283-286.

35. P. Burk, "Jammin' on the Web: A New Client/Server Architecture for Multi-User Musical Performance," in Proceedings of the International Computer Music Conference (ICMC, 2000) pp. 117-120.

36. M. Mühlhäuser, M. Welzl, J. Borchers and R. Gutkas, "GlobeMusic: The Internet Scale of eMusicMaking," Proceedings of the WEDLMUSIC-Web Delivery of Music-Conference (IEEE Computer Society Press, 2001) pp. 131-138.

37. S. Jordà and A. Barbosa, "Computer Supported Cooperative Music: Overview of Research Work and Projects at the Audiovisual Institute-UPF," Proceedings of MOSART Workshop on Current Research Directions in Computer Music (MOSART IHP Network, 2001) pp. 92-97.

38. Burk [35].

39. A. Tanaka, $M P 3 Q(2000)$. For more information, see $<$ http://fals.ch/Dx/atau/mp3q/>.

40. A. Barbosa and M. Kaltenbrunner, "Public Sound Objects: A Shared Musical Space on the Web," in Proceedings of International Conference on Web Delivering of Music 2002, Darmstadt, Germany (IEEE Computer Society Press, 2002) pp. 9-15.
41. T. Rodden, "A Survey of CSCW Systems," Interacting with Computers 3, No. 3 (St. Louis, MO: Butterworth-Heinemann, 1991) pp. 319-353.

42. P. Curtis, "Mudding: Social Phenomena in TextBased Virtual Realities," Proceedings of the 1992 Conference on the Directions and Implications of Advanced Computing (Berkeley, CA: 1992) pp. 1-21.

Manuscript received 20 December 2002.

Álvaro Barbosa is a researcher with the Music Technology Group at Pompeu Fabra University in Barcelona, Spain. He holds an undergraduate degree in electronics and telecommunications engineering from Aveiro University in Portugal and a postgraduate degree in digital arts from the Portuguese Catholic University in Porto. His work as an experimental sonic artist and researcher has been featured in several artistic and scientific events in Portugal and abroad ( Jubileu 2000, Olhares de Outono, MOSART, Wedelmusic, $I C M C)$. His recent research is focused on the study and development of experimental systems for network music, currently under the framework of a preliminary project entitled Public Sound Objects.

\section{Leonardo Electronic Almanac}

The Leonardo Electronic Almanac is issuing an open call for papers. The LEA Editorial Board seeks proposals for the following:

Theoretical Discussions: original essays documenting research and critical commentary in areas of discussion such as nanotechnology, cyberart, cyberfeminism, hypertext, robotics, bio-art, artificial life and genetics. This list is by no means exhaustive, and proposals need not be limited to these areas.

Artists' Statements and Gallery Commissions: international artists are encouraged to submit statements or proposals for exhibiting new media artwork. Curators are welcome to propose thematic exhibitions.

LEA encourages international artists, academics, researchers and students to submit proposals for consideration. We particularly encourage authors outside North America and Europe to send proposals for articles.

Proposals should include:

- a 150-300 word abstract or synopsis detailing subject matter

- a brief bio (and list of prior works for reference)

- names of collaborators (if suggesting a thematic issue or curated gallery)

- any related URLs

- contact details.

Please send proposals or queries to:

Nisar Keshvani

Editor-in-Chief

Leonardo Electronic Almanac

$<$ http://mitpress2.mit.edu/e-journals/LEA $>$

E-mail: <lea@mitpress.mit.edu $>$ 